Bull. Korean Math. Soc. 52 (2015), No. 5, pp. 1535-1547

http://dx.doi.org/10.4134/BKMS.2015.52.5.1535

\title{
SOME RESULTS ON CONCIRCULAR VECTOR FIELDS AND THEIR APPLICATIONS TO RICCI SOLITONS
}

\author{
BANG-YEN CHEN
}

Abstract. A vector field on a Riemannian manifold $(M, g)$ is called concircular if it satisfies $\nabla_{X} v=\mu X$ for any vector $X$ tangent to $M$, where $\nabla$ is the Levi-Civita connection and $\mu$ is a non-trivial function on $M$. A smooth vector field $\xi$ on a Riemannian manifold $(M, g)$ is said to define a Ricci soliton if it satisfies the following Ricci soliton equation:

$$
\frac{1}{2} \mathcal{L}_{\xi} g+R i c=\lambda g,
$$

where $\mathcal{L}_{\xi} g$ is the Lie-derivative of the metric tensor $g$ with respect to $\xi$, Ric is the Ricci tensor of $(M, g)$ and $\lambda$ is a constant. A Ricci soliton $(M, g, \xi, \lambda)$ on a Riemannian manifold $(M, g)$ is said to have concircular potential field if its potential field $\xi$ is a concircular vector field.

In the first part of this paper we determine Riemannian manifolds which admit a concircular vector field. In the second part we classify Ricci solitons with concircular potential field. In the last part we prove some important properties of Ricci solitons on submanifolds of a Riemannian manifold equipped with a concircular vector field.

\section{Introduction}

A. Fialkow introduced in [13] the notion of concircular vector fields on a Riemannian manifold $M$ as vector fields which satisfy

$$
\nabla_{X} v=\mu X, \quad X \in T M
$$

where $\nabla$ denotes the Levi-Civita connection, $T M$ the tangent bundle of $M$ and $\mu$ a non-trivial function on $M$. Concircular vector fields also known as geodesic fields in literature since integral curves of such vector fields are geodesics (see e.g. [18]). A concircular vector field $v$ is called a concurrent vector field if the function $\mu$ in Eq. (1) is one (see, for instance, $[2,8,9]$ ). The notion of concircular vector fields can be extended naturally to concircular vector fields in pseudo-Riemannian manifolds.

Received August 12, 2014.

2010 Mathematics Subject Classification. 53C25, 53C40.

Key words and phrases. concircular vector field, Ricci soliton, submanifolds, Einstein manifold, concircular potential field, concurrent vector field, concircular curvature tensor. 
Concircular vector fields appeared in the study of concircular mappings, i.e., conformal mappings preserving geodesic circles [20]. Such vector fields also play an important role in the theory of projective and conformal transformations. Concircular vector fields have interesting applications in physics as well, in particular in general relativity, e.g., trajectories of time-like concircular fields in the de Sitter model determine the world lines of receding or colliding galaxies satisfying the Weyl hypothesis (cf. [19]). Also it was proved recently by the author in [4] that a Lorentzian manifold is a generalized Robertson-Walker spacetime if and only if it admits a timelike coincircular vector field.

A smooth vector field $\xi$ on a Riemannian manifold $(M, g)$ is said to define a Ricci soliton if it satisfies the following Ricci soliton equation:

$$
\frac{1}{2} \mathcal{L}_{\xi} g+R i c=\lambda g
$$

where $\mathcal{L}_{\xi} g$ is the Lie-derivative of the metric tensor $g$ with respect to $\xi$, Ric is the Ricci tensor of $(M, g)$ and $\lambda$ is a constant. We shall denote a Ricci soliton by $(M, g, \xi, \lambda)$. We call the vector field $\xi$ the potential field. A Ricci soliton $(M, g, \xi, \lambda)$ is called shrinking, steady or expanding according to $\lambda>0, \lambda=0$, or $\lambda<0$, respectively. A trivial Ricci soliton is one for which $\xi$ is zero or Killing, in which case the metric is Einsteinian (see, for instance, $[6,7,10,14,16,17]$ ).

Compact Ricci solitons are the fixed points of the Ricci flow:

$$
\frac{\partial}{\partial t} g(t)=-2 \operatorname{Ric}(g(t))
$$

projected from the space of metrics onto its quotient modulo diffeomorphisms and scalings, and often arise as blow-up limits for the Ricci flow on compact manifolds. Further, Ricci solitons model the formation of singularities in the Ricci flow and they correspond to self-similar solutions (cf. e.g. [16]).

A Ricci soliton $(M, g, \xi, \lambda)$ is called gradient if its potential field $\xi$ is the gradient of some function $f$ on $M$. We denote such a gradient Ricci soliton by $(M, g, f, \lambda)$ and call the smooth function $f$ the potential function. For a gradient Ricci soliton $(M, g, f, \lambda)$, the soliton equation can be expressed as

$$
R i c_{f}=\lambda g
$$

where Ric $c_{f}:=$ Ric + Hess $(f)$ is known as the Bakry-Emery curvature. Hence a gradient Ricci soliton has constant Bakry-Emery curvature; a similar role as an Einstein manifold.

A gradient Ricci soliton $(M, g, f, \lambda)$ is called trivial if its potential function $f$ is a constant. It follows from Eq.(2) that trivial gradient Ricci solitons are trivial Ricci solitons automatically. G. Perelman proved in [17] that the Ricci solitons on compact simply connected Riemannian manifolds are gradient Ricci solitons as solutions of Ricci flow.

During the last two decades, the geometry of Ricci solitons has been the focus of attention of many mathematicians. It has become more important 
after G. Perelman applied Ricci solitons to solve the long standing Poincaré conjecture posed in 1904 .

In the first part of this paper we determine Riemannian manifolds which admit a concircular vector field. In the second part we classify Ricci solitons with concircular potential fields. In the last part we prove some important properties of Ricci solitons on submanifolds of a Riemannian manifold equipped with a concircular vector field.

\section{Preliminaries}

\subsection{Basic formulas and definitions for submanifolds}

For general references on Riemannian submanifolds, we refer to $[2,3,5]$.

Let $\left(N^{m}, \tilde{g}\right)$ denote an $m$-dimensional Riemannian manifold and let $\phi$ : $M^{n} \rightarrow N^{m}$ be an isometric immersion from an $n$-dimensional Riemannian manifold $\left(M^{n}, g\right)$ into $\left(N^{m}, \tilde{g}\right)$. Denote by $\nabla$ and $\tilde{\nabla}$ the Levi-Civita connections on $\left(M^{n}, g\right)$ and $\left(N^{m}, \tilde{g}\right)$, respectively.

For vector fields $X, Y$ tangent to $M^{n}$ and $\eta$ normal to $M^{n}$, the formula of Gauss and the formula of Weingarten are given respectively by

$$
\begin{aligned}
& \tilde{\nabla}_{X} Y=\nabla_{X} Y+h(X, Y), \\
& \tilde{\nabla}_{X} \eta=-A_{\eta} X+D_{X} \eta,
\end{aligned}
$$

where $\nabla_{X} Y$ and $h(X, Y)$ are the tangential and the normal components of $\tilde{\nabla}_{X} Y$. Similarly, $-A_{\eta} X$ and $D_{X} \eta$ are the tangential and normal components of $\tilde{\nabla}_{X} \eta$. These two formulas define the second fundamental form $h$, the shape operator $A$, and the normal connection $D$ of $M^{n}$ in the ambient space $N^{m}$.

For a normal vector $\eta \in T_{p}^{\perp} M$ at $p \in M, A_{\eta}$ is a self-adjoint endomorphism of the tangent space $T_{p} M$. The shape operator and the second fundamental form are related by

$$
\tilde{g}(h(X, Y), \eta)=g\left(A_{\eta} X, Y\right) .
$$

The mean curvature vector $H$ of $M^{n}$ in $N^{m}$ is defined by

$$
H=\left(\frac{1}{n}\right) \text { trace } h .
$$

The equations of Gauss and Codazzi are given respectively by

$$
\begin{aligned}
& g(R(X, Y) Z, W)= \tilde{g}(\tilde{R}(X, Y) Z, W)+\tilde{g}(h(X, W), h(Y, Z)) \\
&-\tilde{g}(h(X, Z), h(Y, W)), \\
&(\tilde{R}(X, Y) Z)^{\perp}=\left(\bar{\nabla}_{X} h\right)(Y, Z)-\left(\bar{\nabla}_{Y} h\right)(X, Z),
\end{aligned}
$$

for vectors $X, Y, Z, W$ tangent to $M$ and $\zeta, \eta$ normal to $M$, where $(\tilde{R}(X, Y) Z)^{\perp}$ is the normal component of $\tilde{R}(X, Y) Z$ and $\bar{\nabla} h$ is defined by

$$
\left(\bar{\nabla}_{X} h\right)(Y, Z)=D_{X} h(Y, Z)-h\left(\nabla_{X} Y, Z\right)-h\left(Y, \nabla_{X} Z\right) .
$$


For a function $f$ on a Riemannian manifold $M$, we denote by $\nabla f$ and $H^{f}$ the gradient of $f$ and the Hessian of $f$, respectively. Thus we have

$$
\begin{aligned}
& g(\nabla f, X)=X f, \\
& H^{f}(X, Y)=X Y f-\left(\nabla_{X} Y\right) f .
\end{aligned}
$$

Let $\left\{e_{1}, \ldots, e_{n}\right\}$ be a local orthonormal frame on a Riemannian $n$-manifold $M^{n}$. Denote by $\left\{\omega^{1}, \ldots, \omega^{n}\right\}$ the dual frame of 1 -forms of $\left\{e_{1}, \ldots, e_{n}\right\}$. The connection forms $\omega_{i}^{j}(i, j=1, \ldots, n)$ are defined by

$$
\nabla_{X} e_{i}=\sum_{j=1}^{n} \omega_{i}^{j}(X) e_{j}, \quad i=1, \ldots, n .
$$

From Cartan's structure equations, we have

$$
d \omega^{i}=-\sum_{j=1}^{n} \omega_{j}^{i} \wedge \omega^{j}, \quad i=1, \ldots, n .
$$

A foliation $\mathcal{D}$ on a manifold $M$ is an integrable distribution, i.e., $\mathcal{D}$ is a vector subbundle of the tangent bundle $T M$ such that, for any vector fields $X, Y$ in $\mathcal{D}$, the Lie bracket $[X, Y]$ takes values in $\mathcal{D}$ as well. A foliation $\mathcal{D}$ on a Riemannian manifold $M$ is called totally umbilical, if every leaf of $\mathcal{D}$ is a totally umbilical submanifold of $M$. If, in addition, the mean curvature vector of every leaf is parallel in the normal bundle, then $\mathcal{D}$ is called a spherical foliation. In this case, leaves of $\mathcal{D}$ are extrinsic spheres of $M$. If leaves of a foliation $\mathcal{D}$ are totally geodesic submanifolds, $\mathcal{D}$ is called a totally geodesic foliation (cf. e.g., $[3,5])$.

\section{Riemannian manifolds with concircular vector fields}

First, we give the following examples of Riemannian manifolds endowed with concircular vector fields.

Example 3.1. Let $I$ be an open interval of the real line $\mathbf{R}$ and let $\varphi(s), s \in I$, be a function on $I$ which is nowhere zero. Consider a warped product manifold of the form:

$$
I \times \times_{\varphi(s)} F,
$$

where $F$ is a Riemannian manifold. The metric tensor $g$ of $I \times \varphi(s) F$ is given by $g=d s^{2}+\varphi^{2}(s) g_{F}$, where $g_{F}$ is the metric tensor of the second factor $F$. Consider the vector field given by

$$
v=\varphi(s) \frac{\partial}{\partial s} .
$$

It follows from Proposition 4.1 of [3, page 79] that the vector field $v$ satisfies Eq. (1) with $\mu=\varphi^{\prime}(s)$. Thus $v$ is a concircular vector field.

The next result implies that Example 3.1 provides all Riemannian manifolds which admit a nowhere zero concircular vector field. 
Theorem 3.1. If $M$ is a Riemannian n-manifold which admits a nowhere zero concircular vector field, then $M$ is locally a warped product $I \times_{\varphi(s)} F$, where $\varphi(s)$ is a nowhere vanishing function and $F$ is a Riemannian $(n-1)$-manifold.

Proof. Assume that $v$ is a concircular vector field on a Riemannian manifold such that $v$ is nowhere zero. Let us put

$$
v=\varphi e_{1},
$$

where $e_{1}$ is a unit vector field in the direction of $v$. Extend $e_{1}$ to an orthonormal frame $e_{1}, \ldots, e_{n}$ on $M$. It follows from (1) and a direct computation that

$$
\begin{aligned}
R\left(e_{i}, v\right) v & =\nabla_{e_{i}} \nabla_{v} v-\nabla_{v} \nabla_{e_{i}} v-\nabla_{\left[e_{i}, v\right]} v \\
& =\left(e_{i} \mu\right) v-(v \mu) e_{i}, \quad i=2, \ldots, n,
\end{aligned}
$$

where $\mu$ is defined by (1). From (17) and $\left\langle R\left(e_{i}, v\right) v, v\right\rangle=0$, we get

$$
e_{2} \mu=\cdots=e_{n} \mu=0 \text {. }
$$

Thus the gradient $\nabla \mu$ is parallel to $v$.

From (1) with $X=e_{1}$ and (16) we find

$$
\mu e_{1}=\nabla_{e_{1}}\left(\varphi e_{1}\right)=\left(e_{1} \varphi\right) e_{1}+\varphi \nabla_{e_{1}} e_{1},
$$

which gives

$$
e_{1} \varphi=\mu, \quad \nabla_{e_{1}} e_{1}=0 .
$$

From the second equation in (19) we know that the integral curves of $e_{1}$ are geodesics in $M$. Therefore the distribution $\mathcal{D}_{1}=\operatorname{Span}\left\{e_{1}\right\}$ is a totally geodesic foliation. Let us put $\mathcal{D}_{2}=\operatorname{Span}\left\{e_{2}, \ldots, e_{n}\right\}$.

From (16) and (1) with $X=e_{i}$ with $i=2, \ldots, n$, we find

$$
\mu e_{i}=\nabla_{e_{i}}\left(\varphi e_{1}\right)=\left(e_{i} \varphi\right) e_{1}+\varphi \nabla_{e_{i}} e_{1},
$$

which implies

$$
\begin{aligned}
& e_{2} \varphi=\cdots=e_{n} \varphi=0, \\
& \varphi \nabla_{e_{i}} e_{1}=\mu e_{i} .
\end{aligned}
$$

It follows from (12) and (21) that

$$
\omega_{i}^{1}\left(e_{j}\right)=\frac{\mu}{\varphi} \delta_{i j}, \quad 2 \leq i, j \leq n .
$$

From (22) we conclude that $\mathcal{D}_{2}$ is an integrable distribution whose leaves are totally umbilical in $M$. Moreover, the mean curvature of leaves of $\mathcal{D}_{2}$ are given by $\mu / \varphi$. Since leaves of $\mathcal{D}_{2}$ are hypersurfaces, it follows from (18) and (20) that the mean curvature vector fields of leaves of $\mathcal{D}_{2}$ are parallel in the normal bundle in $M$. Thus $\mathcal{D}_{2}$ is a spherical foliation. Consequently, by a result of [15] (or Theorem 4.4 of $[3$, page 90$]$ ) we conclude that $M$ is locally a warped product 
$I \times_{f(s)} F$, where $f(s)$ is a function on $I, \partial / \partial s=e_{1}$, and $F$ is a Riemannian $(n-1)$-manifold. Therefore the sectional curvature of $M$ satisfies

$$
K\left(e_{1}, X\right)=-\frac{f^{\prime \prime}(s)}{f(s)}
$$

for any unit vector $X$ orthogonal to $e_{1}$.

On the other hand, it follows from (16) and (17) that we also have

$$
\varphi K\left(e_{1}, X\right)=-v \mu=-\mu^{\prime}(s)
$$

for any unit vector $X$ orthogonal to $e_{1}$. Thus, after combining (24) with (19) and (23), we obtain

$$
\frac{f^{\prime \prime}(s)}{f(s)}=\frac{\mu^{\prime}(s)}{\varphi}=\frac{\varphi^{\prime \prime}(s)}{\varphi(s)} .
$$

Thus if we choose $f(s)=\varphi(s)$, then $M$ is locally a warped product $I \times_{\varphi(s)} F$. A direct computation yields $\nabla_{X}\left(\varphi(s) \frac{\partial}{\partial s}\right)=\varphi^{\prime}(s) X$ for $X$ tangent to $I \times_{f(s)} F$.

\section{Concircular vector fields on real space forms}

In this section we determine all concircular vector fields on Riemannian manifolds of constant curvature.

First we give the following necessary and sufficient condition for a gradient vector field on a Riemannian manifold to be concircular.

Lemma 4.1. Let $f$ be a function on a Riemannian manifold $M$. Then the gradient $\nabla f$ of $f$ is a concircular vector field if and only if the Hessian $H^{f}$ of $f$ satisfies

$$
H^{f}(X, Y)=\mu g(X, Y)
$$

for $X, Y$ tangent to $M$, where $\mu$ is the function on $M$. Moreover, in such case the function $\mu$ satisfies Eq. (1) with $v=\nabla f$.

Proof. Let $f$ be a function of a Riemannian manifold $M$. Assume that the Hessian of $f$ satisfies (25). Then we have

$$
\begin{aligned}
g(X, \mu Y) & =X Y f-\nabla_{X} Y f \\
& =X\langle Y, \nabla f\rangle-\left\langle\nabla_{X} Y, \nabla f\right\rangle \\
& =\left\langle Y, \nabla_{X}(\nabla f)\right\rangle
\end{aligned}
$$

for vector fields $X, Y$ tangent to $M$. Thus we obtain $\nabla_{X}(\nabla f)=\mu X$, which implies that $v=\nabla f$ is a concircular vector field satisfying Eq. (1).

The converse can be verified in a similar way.

The following result classifies all concircular vector fields on $\mathbb{E}^{n}$.

Proposition 4.2. Let $v$ be a nonzero vector field on the Euclidean $n$-space $\mathbb{E}^{n}$. Then $v$ is a concircular vector field if and only if only if $v=b \mathbf{x}$, where $b$ is $a$ nonzero constant and $\mathbf{x}$ is a concurrent vector field. 
Proof. If $v$ is a concircular vector field on a Riemannian manifold, then it follows from (1) and a direct computation that

$$
\begin{aligned}
R(X, v) v & =\nabla_{X} \nabla_{v} v-\nabla_{v} \nabla_{X} v-\nabla_{[X, v]} v \\
& =(X \mu) v-(v \mu) X
\end{aligned}
$$

for any vector field $X$ perpendicular to $v$, where $\mu$ is defined by (1). It follows from (27) that the gradient $\nabla \mu$ is parallel to $v$.

If $M$ is the Euclidean $n$-space $\mathbb{E}^{n}$, we have $R=0$. Hence we obtain from (27) that $X v=v \mu=0$, which implies that $\mu$ is a nonzero constant, say $b$. Consequently, $\mathbf{x}=v / b$ is a concurrent vector field.

The converse is trivial.

The next result determines all concircular vector fields on the unit $n$-sphere.

Proposition 4.3. Let $\left\{u_{1}, \ldots, u_{n}\right\}$ be an isothermal coordinate system on $S^{n}(1)$ so that the metric tensor of $S^{n}(1)$ is given by

$$
g=\frac{4}{\left(1+\sum_{j=1}^{n} u_{j}^{2}\right)^{2}} \sum_{i=1}^{n} d u_{i}^{2} .
$$

Then a vector field $v$ on $S^{n}(1)$ is concircular if and only if, up to translations of $u_{1}, \ldots, u_{n}, v$ is a gradient vector field given by $v=-\nabla \mu$, where

$$
\mu=\frac{1-\sum_{i=1}^{n} u_{i}^{2}}{2+2 \sum_{j=1}^{n} u_{j}^{2}} .
$$

Moreover, $\mu$ is exactly the function satisfying (1).

Proof. Let $\left\{u_{1}, \ldots, u_{n}\right\}$ be the isothermal coordinates so that the metric tensor of $S^{n}(1)$ is given by Eq. (28). If $v$ is a concircular vector field of $S^{n}(1)$, then (27) holds.

Since $S^{n}(1)$ is of constant curvature one, we also have

$$
R(X, v) v=g(v, v) X-g(X, v) v
$$

for $X$ perpendicular to $v$. Thus we find from (27) and (30) that

$$
X \mu=-g(X, v), \quad v \mu=-g(v, v) .
$$

Hence $v=\nabla f$ is a gradient field with $f=-\mu$.

On the other hand, it follows from a direct computation that the Levi-Civita connection of (28) satisfies

$$
\begin{aligned}
& \nabla_{\partial_{u_{i}}} \partial_{u_{i}}=\frac{-2}{1+\sum_{i=1}^{n} u_{i}^{2}}\left\{u_{i} \partial_{u_{i}}-\sum_{j \neq i} u_{j} \partial_{u_{j}}\right\}, \\
& \nabla_{\partial_{u_{i}}} \partial_{u_{j}}=\frac{-2}{1+\sum_{i=1}^{n} u_{i}^{2}}\left\{u_{i} \partial_{u_{j}}+u_{j} \partial_{u_{i}}\right\}, \quad 1 \leq i \neq j \leq n,
\end{aligned}
$$

where $\partial_{u_{i}}=\partial / \partial u_{i}, i=1, \ldots, n$. 
From $f=-\mu$, Eqs. (11) and (32) we find

$$
\begin{aligned}
& H^{f}\left(\partial_{u_{i}}, \partial_{u_{i}}\right)=-\frac{\partial^{2} \mu}{\partial^{2} u_{i}}-\frac{2}{1+\sum_{i=1}^{n} u_{i}^{2}}\left\{u_{i} \frac{\partial \mu}{\partial u_{i}}-\sum_{j \neq i} u_{j} \frac{\partial \mu}{\partial u_{j}}\right\}, \\
& H^{f}\left(\partial_{u_{i}}, \partial_{u_{j}}\right)=-\frac{\partial^{2} \mu}{\partial u_{i} \partial u_{j}}-\frac{2}{1+\sum_{i=1}^{n} u_{i}^{2}}\left\{u_{j} \frac{\partial \mu}{\partial u_{i}}+u_{i} \frac{\partial \mu}{\partial u_{j}}\right\}
\end{aligned}
$$

for $1 \leq i \neq j \leq n$. After combining Eq. (33) with Eq. (25) of Lemma 4.1, we get

$$
\begin{aligned}
& \frac{\partial^{2} \mu}{\partial u_{i} \partial u_{j}}=\frac{-2}{1+\sum_{i=1}^{n} u_{i}^{2}}\left\{u_{j} \frac{\partial \mu}{\partial u_{i}}+u_{i} \frac{\partial \mu}{\partial u_{j}}\right\} \\
& \frac{\partial^{2} \mu}{\partial^{2} u_{i}}=\frac{-4 \mu}{\left(1+\sum_{i=1}^{n} u_{i}^{2}\right)^{2}}-\frac{2}{1+\sum_{i=1}^{n} u_{i}^{2}}\left\{u_{i} \frac{\partial \mu}{\partial u_{i}}-\sum_{j \neq i} u_{j} \frac{\partial \mu}{\partial u_{j}}\right\}
\end{aligned}
$$

for $1 \leq i \neq j \leq n$.

After solving system (34)-(35) via long computation and by applying suitable translations on $u_{i}$, we obtain

$$
\mu=\frac{1-\sum_{i=1}^{n} u_{i}^{2}}{2+2 \sum_{j=1}^{n} u_{j}^{2}} .
$$

Consequently, by Lemma 4.1, the concircular vector field $v$ is given by

$$
v=\frac{1}{2} \sum_{j=1}^{n} u_{j} \partial u_{j} .
$$

Conversely, by applying (28), (32) and a straight-forward computation, we obtain $\nabla_{V} v=\mu V$ for $V$ tangent to $S^{n}(1)$.

Similarly, we have the following result for the hyperbolic $n$-space $H^{n}(-1)$ with constant curvature -1 .

Proposition 4.4. Let $\left\{v_{1}, \ldots, v_{n}\right\}$ be an isothermal coordinates on $H^{n}(-1)$ so that the metric tensor of $H^{n}(-1)$ is

$$
g=\frac{4}{\left(1-\sum_{j=1}^{n} v_{j}^{2}\right)^{2}} \sum_{i=1}^{n} d v_{i}^{2} .
$$

Then a vector field $v$ on $H^{n}(-1)$ is concircular if and only if, up to translations of $v_{1}, \ldots, v_{n}, v$ is a gradient vector field given by $v=-\nabla \mu$, where

$$
\mu=\frac{1+\sum_{i=1}^{n} v_{i}^{2}}{2-2 \sum_{j=1}^{n} v_{j}^{2}} .
$$

Moreover, $\mu$ is exactly the function satisfying (1).

Proof. This can be done in the same way as Proposition 4.3. 


\section{Ricci solitons with concircular potential field}

Next, we apply our previous results to classify Ricci solitons with concircular potential field.

Theorem 5.1. A Ricci soliton $(M, g, v, \lambda)$ on a Riemannian $n$-manifold $(M, g)$ with $n \geq 3$ has concircular potential field $v$ if and only if the following three conditions hold:

(a) The function $\mu$ in (1) is a nonzero constant, say b;

(b) $\lambda=b$;

(c) $M$ is an open portion of a warped product manifold $I \times_{b s+c} F$, where $I$ is an open interval with arc-length $s, c$ is a constant, and $F$ is an Einstein $(n-1)$-manifold whose Ricci tensor satisfies $\operatorname{Ric}_{F}=(n-2) b^{2} g_{F}, g_{F}$ being the metric tensor of $F$.

Proof. Assume that $(M, g, v, \lambda)$ is a Ricci soliton on a Riemannian $n$-manifold with a concircular potential field $v$. Then Eq. (1) holds, which implies

$$
\left(\mathcal{L}_{v} g\right)(X, Y)=g\left(\nabla_{X} v, Y\right)+g\left(\nabla_{Y} v, X\right)=2 \mu g(X, Y)
$$

for any $X, Y$ tangent to $M$. Combining (2) and (40) gives

$$
\operatorname{Ric}(X, Y)=(\lambda-\mu) g(X, Y)
$$

which shows that $M$ is an Einstein manifold. Since $n \geq 3$ and $M$ is Einsteinian, $M$ has constant scalar curvature. So $\lambda-\mu$ is constant. Thus the function $\mu$ is also a nonzero constant, say $b$. This gives statement (a). In particular, we have

$$
\nabla_{X} v=b X, \quad X \in T M .
$$

Since $\mu=b$ is constant, (27) implies $R(X, v) v=0$ for $X$ orthogonal to $v$. Hence $\operatorname{Ric}(v, v)=0$. Therefore $M$ is Ricci-flat. Consequently, we obtain from (41) that $\lambda=b$. This gives statement (b).

It follows from Theorem 3.1 that $M$ is locally a warped product $I \times_{\varphi(s)} F$ for some function $\varphi(s)$ and Riemannian $(n-1)$-manifold $F$. Moreover, from statement (a) and the proof of Theorem 3.1 we also have $\varphi^{\prime}(s)=\mu=b$. Thus $\varphi=b s+c$ for some constant $c$. Hence $M$ is an open part of a warped product manifold $I \times{ }_{b s+c} F$.

Since $M$ is Ricci-flat, (41) yields $\lambda=\mu=b$. Now, it follows from formula (9.109) of [1, page 267] that the second factor $F$ of $I \times_{b s+c} F$ is an Einstein manifold satisfying $\operatorname{Ric}_{F}=(n-2) b^{2} g_{F}$.

The converse can be verified by direct computation.

An easy consequence of Theorem 5.1 is the following.

Corollary 5.2. The only Riemannian manifold of constant sectional curvature admitting a Ricci soliton with concircular potential field is a Euclidean space.

Proof. Follows from Proposition 4.3, Proposition 4.4 and Theorem 5.1(a). 
Associated with the Ricci tensor on a Riemannian $n$-manifold, let $Q$ denote the $(1,1)$-tensor defined by $\langle Q(X), Y\rangle=\operatorname{Ric}(X, Y)$.

The Weyl conformal curvature tensor $W$ is of type $(1,3)$ is defined by

$$
\begin{aligned}
W(X, Y) Z= & R(X, Y) Z+\frac{1}{n}\{\operatorname{Ric}(X, Z) Y-\operatorname{Ric}(Y, Z) X+\langle X, Z\rangle Q Y \\
& -\langle Y, Z\rangle Q X\}-\frac{2 \tau}{n(n+1)}\{\langle X, Z\rangle Y-\langle Y, Z\rangle X\},
\end{aligned}
$$

where $\tau$ is the scalar curvature.

It is well-known that the Weyl conformal curvature tensor $W$ vanishes identically when $\operatorname{dim} M=3$. A Riemannian $n$-manifold with $n \geq 4$ is called conformally flat if its Weyl conformal curvature tensor vanishes identically.

Another easy consequence of Theorem 5.1 is the following.

Corollary 5.3. The only conformally flat manifold of dimension $\geq 4$ which admits a Ricci soliton with concircular potential field is the Euclidean space.

Proof. It follows immediately from (43) that the only conformally flat, Einstein manifolds of dimension $\geq 4$ are Riemannian manifolds of constant curvature. Hence we obtain this corollary from Corollary 5.2.

The concircular curvature tensor $Z$ of type $(1,3)$ on a Riemannian manifold is defined by

$$
Z(X, Y) Z=R(X, Y) Z-\frac{2 \tau}{n(n+1)}\{g(X, Z) Y-g(Y, Z) X\} .
$$

A Riemannian manifold is called concircularly flat if its concircular curvature tensor $Z$ vanishes identically.

Similarly, Theorem 5.1 also implies the following.

Corollary 5.4. The only concircularly flat manifold which admits a Ricci soliton with concircular potential field is the Euclidean space.

\section{Ricci solitons on Riemannian submanifolds arisen from concircular vector fields}

Finally, in this last section we prove some properties of Ricci solitons on submanifolds of Riemannian manifolds equipped with a concircular vector field. These extend some results of [8].

We make the following.

Assumption. $\left(N^{m}, \tilde{g}\right)$ is a Riemannian m-manifold endowed with a concircular vector field $v$.

For a Riemannian submanifold $\left(M^{n}, g\right)$ of $\left(N^{m}, \tilde{g}\right)$, we denote by $v^{T}$ and $v^{\perp}$ the tangential and normal components on $M^{n}$ of the concircular vector field $v$, respectively. As before, let $h, A$ and $D$ denote the second fundamental form, the shape operator and the normal connection of the submanifold, respectively. 
Theorem 6.1. A submanifold $M^{n}$ in $N^{m}$ admits a Ricci soliton $\left(M^{n}, g, v^{T}, \lambda\right)$ if and only if the Ricci tensor of $M^{n}$ satisfies

$$
\operatorname{Ric}(X, Y)=(\lambda-\mu) g(X, Y)-\tilde{g}\left(h(X, Y), v^{\perp}\right)
$$

for any $X, Y$ tangent to $M^{n}$.

Proof. Let $\phi: M^{n} \rightarrow N^{m}$ denote the isometric immersion. We have

$$
v=v^{T}+v^{\perp} .
$$

Since $v$ is a concircular vector field on $N^{m}$, it follows from (1), (46) and formulas of Gauss and Weingarten that

$$
\mu X=\nabla_{X} v^{T}+h\left(X, v^{T}\right)-A_{v^{\perp}} X+D_{X} v^{\perp}
$$

for any $X$ tangent to $M^{n}$. By comparing the tangential and normal components from (47) we obtain

$$
\nabla_{X} v^{T}=A_{v^{\perp}} X+\mu X
$$

From the definition of Lie derivative and (48) we find

$$
\left(\mathcal{L}_{v^{T}} g\right)(X, Y)=2 \mu g(X, Y)+2 \tilde{g}\left(h(X, Y), v^{\perp}\right)
$$

for $X, Y$ tangent to $M^{n}$. Consequently, by applying (2) and (49), we conclude that $\left(M^{n}, g, v^{T}, \lambda\right)$ is a Ricci soliton if and only if

$$
\operatorname{Ric}(X, Y)+\mu g(X, Y)+\tilde{g}\left(h(X, Y), v^{\perp}\right)=\lambda g(X, Y),
$$

which is nothing but (45).

A Riemannian submanifold $M^{n}$ is called $\eta$-umbilical with respect to a normal vector field $\eta$ if its shape operator satisfies $A_{\eta}=\varphi I$, where $\varphi$ is a function on $M^{n}$ and $I$ is the identity map.

The following two results are immediate consequences of Theorem 6.1.

Corollary 6.2. A Ricci soliton $\left(M^{n}, g, v^{T}, \lambda\right)(n \geq 3)$ on a submanifold $M^{n}$ in $N^{m}$ is trivial if and only if $M^{n}$ is $v^{\perp}$-umbilical.

Corollary 6.3. Every Ricci soliton $\left(M^{n}, g, v^{T}, \lambda\right)$ on a totally umbilical submanifold $M^{n}$ of $N^{m}$ is a trivial Ricci soliton.

Following [3], the scalar curvature $\tau$ of $\left(M^{n}, g\right)$ is defined to be

$$
\tau=\sum_{1 \leq i<j \leq n} K\left(e_{i}, e_{j}\right),
$$

where $\left\{e_{1}, \ldots, e_{n}\right\}$ is an orthonormal frame of $M^{n}$.

Another easy application of Theorem 6.1 is the following.

Corollary 6.4. If $\left(M^{n}, g, v^{T}, \lambda\right)$ is a Ricci soliton on a minimal submanifold $M^{n}$ in $N^{m}$, then the scalar curvature $\tau$ of $M^{n}$ is given by $n(\lambda-\mu) / 2$. 
Proof. Assume that $\left(M^{n}, g, v^{T}, \lambda\right)$ is a Ricci soliton on a submanifold $M^{n}$ in $N^{m}$. Then it follows from Theorem 6.1 that the Ricci tensor satisfies

$$
\operatorname{Ric}(X, Y)=(\lambda-\mu) g(X, Y)-\tilde{g}\left(h(X, Y), v^{\perp}\right)
$$

for $X, Y$ tangent to $M^{n}$. If $M^{n}$ is minimal, then $\tilde{g}\left(H, v^{\perp}\right)=0$. Hence (52) gives $\sum_{i=1}^{n} \operatorname{Ric}\left(e_{i}, e_{i}\right)=n(\lambda-\mu)$. Thus $M^{n}$ has constant scalar curvature $n(\lambda-\mu) / 2$.

Corollary 6.5. Let $R^{n+1}(c)$ be a Riemannian manifold of constant curvature $c$ equipped with a concircular vector field $v$. If $\left(M^{n}, g, v^{T}, \lambda\right)$ is a Ricci soliton on a hypersurface of $M^{n}$ of $R^{n+1}(c)$, then $M^{n}$ has at most two distinct principal curvatures given by

$$
\kappa_{1}, \kappa_{2}=\frac{n \alpha+\rho \pm \sqrt{(n \alpha+\rho)^{2}-4(\lambda-\mu-(n-1) c)}}{2},
$$

where $\alpha$ is the mean curvature, i.e., $H=\alpha N$ and $\rho=\langle N, v\rangle$ with $N$ being a unit normal vector field.

Proof. Under the hypothesis, assume that $\left(M^{n}, g, v^{T}, \lambda\right)$ is a Ricci soliton on a hypersurface of $M^{n}$ of $R^{n+1}(c)$, where $v^{T}$ denotes the tangential component of the concircular vector field $v$. Let $\left\{e_{1}, \ldots, e_{n}\right\}$ be an orthonormal frame on $M^{n}$ such that $e_{1}, \ldots, e_{n}$ are eigenvectors of the shape operator $A_{N}$. Then we have

$$
A_{N} e_{i}=\kappa_{i} e_{i}, \quad i=1, \ldots, n .
$$

From equation (7) of Gauss we obtain

$$
\begin{aligned}
\operatorname{Ric}(X, Y)= & (n-1) c g(X, Y)+n \tilde{g}(h(X, Y), H) \\
& -\sum_{i=1}^{n} \tilde{g}\left(h\left(X, e_{i}\right), h\left(Y, e_{i}\right)\right),
\end{aligned}
$$

where $\tilde{g}$ denotes the metric of $R^{n+1}(c)$.

It follows from (54), (55) and Theorem 6.1 that $\left(M^{n}, g, v^{T}, \lambda\right)$ is a Ricci soliton if and only if we have

$$
\left\{(n-1) c+\left(n \alpha-\kappa_{j}\right) \kappa_{i}\right\} \delta_{i j}=(\lambda-\mu) \delta_{i j}-\rho \kappa_{i} \delta_{i j},
$$

where $\delta_{i j}$ is the Kronecker delta. Equation (56) is equivalent to

$$
\kappa_{i}^{2}-(n \alpha+\rho) \kappa_{i}+\lambda-\mu-(n-1) c=0, \quad i=1, \ldots, 0,
$$

which implies the proposition

Remark 6.6. Results in this section extend some results obtained in [8].

Remark 6.7. Ricci solitons on Riemannian submanifolds have also been studied in $[10,11,12]$ by J. T. Cho and M. Kimura from a different viewpoint. They proved several interesting results on Ricci solitons on submanifolds; however their potential fields of the Ricci solitons are quite different from ours. 
Acknowledgements. This work is supported by NPST strategic technologies program number (13-MAT1813-02) in the Kingdom of Saudi Arabia.

\section{References}

[1] A. Besse, Einstein Manifolds, Springer-Verlag, Berlin, 1987.

[2] B.-Y. Chen, Geometry of Submanifolds, Marcel Dekker, New York, 1973.

[3] _ Pseudo-Riemannian Geometry, $\delta$-invariants and Applications, World Scientific, Hackensack, NJ, 2011.

[4] _ A simple characterization of generalized Robertson-Walker spacetimes, Gen. Relativity Gravitation 46 (2014), no. 12, Art. 1833, 5 pp.

[5] _ , Total Mean Curvature and Submanifolds of Finite Type, 2nd Edition, World Scientific, Hackensack, NJ, 2015.

[6] B.-Y. Chen and S. Deshmukh, Geometry of compact shrinking Ricci solitons, Balkan J. Geom. Appl. 19 (2014), no. 1, 13-21.

[7] _ Classification of Ricci solitons on Euclidean hypersurfaces, Internat. J. Math. 25 (2014), no. 11, 1450104, 22 pp.

[8] _ Ricci solitons and concurrent vector fields, Balkan J. Geom. Appl. 20 (2015), no. $1,14-25$.

[9] B.-Y. Chen and K. Yano, On submanifolds of submanifolds of a Riemannian manifold, J. Math. Soc. Japan 23 (1971), no. 3, 548-554.

[10] J. T. Cho and M. Kimura, Ricci solitons and real hypersurfaces in a complex space form, Tohoku Math. J. (2) 61 (2009), no. 2, 205-212.

[11] _ Ricci solitons of compact real hypersurfaces in Kähler manifolds, Math. Nachr. 284 (2011), no. 11-12, 1385-1393.

[12] _ Ricci solitons on locally conformally flat hypersurfaces in space forms, J. Geom. Phys. 62 (2012), no. 8, 1882-1891.

[13] A. Fialkow, Conformals geodesics, Trans. Amer. Math. Soc. 45 (1939), no. 3, 443-473.

[14] R. S. Hamilton, The formation of singularities in the Ricci flow, Surveys in differential geometry, Vol. II (Cambridge, MA, 1993), 7-136, Int. Press, Cambridge, MA, 1995.

[15] S. Hiepko, Eine innere Kennzeichnung der verzerrten Produkte, Math. Ann. 241 (1979), no. 3, 209-215.

[16] J. Morgan and G. Tian, Ricci Flow and the Poincaré Conjecture, Clay Mathematics Monographs, 5, Cambridge, MA, 2014.

[17] G. Perelman, The Entropy Formula For The Ricci Flow And Its Geometric Applications, arXiv math/0211159.

[18] Ya. L. Šapiro, Geodesic fields of directions and projective path systems, Mat. Sb. N.S. 36 (78) (1955), 125-148.

[19] H. Takeno, Concircular scalar field in spherically symmetric space-times I, Tensor 20 (1967), no. 2, 167-176.

[20] K. Yano, Concircular geometry. I. Concircular transformations, Proc. Imp. Acad. Tokyo 16 (1940), 195-200.

Department of Mathematics

Michigan State University

619 Red CEdAR RoAd

EAST LANSing, MI 48824-1027, USA

E-mail address: bychen@math.msu.edu 